\title{
A new interpretation of the Shimura curve with discriminant 6 in terms of Picard modular forms
}

\author{
Maria Petkova and Hironori Shiga
}

Nov. 22., 2010

\begin{abstract}
In this paper we give a realization of the Shimura curve for the quaternion algebra over $\boldsymbol{Q}$ with discriminant 6 as a quotient space of the complex upper half plane by the triangle group $\Delta(3,6,6)$. That is given by the Schwarz map for the Gauss hypergepmetric differential equation $E\left(\frac{1}{6}, \frac{1}{3}, \frac{2}{3}\right)$. The corresponding abelian surfaces are obtained as an isogenous components of the Jacobi varieties of the Picard curves $C(s): w^{3}=z(z-1)\left(z-\frac{1}{2}(1-s)\right)\left(z-\frac{1}{2}(1+s)\right)$.
\end{abstract}

\section{Introduction}

A Shimura curve corresponding to a quaternion algebra $B=\left(\frac{a, b}{F}\right)$ is a 1-dimensional modular variety embedded in a moduli space $\mathfrak{S}_{g}$ of abelian varieties $\{A\}$ of a certain dimension $g$ with generic endmorphism structure $B=\operatorname{End}_{0} A$. We can extract this Shimura curve with its modular group action as a pair of the upper half plane $\boldsymbol{H}$ and a group $G \subset S L(2, \boldsymbol{R})$ so called a norm 1 group of $B$.

In [Voi] we find how we can realize the Shimura curve with discriminant 6 in the projective space generated by the modular forms on $\boldsymbol{H}$ with respect to $G$. Originally this description is given by A. Kurihara [Kur] .

A new approach was proposed in [Pet] standing on the frame work of Picard modular forms for the group $U\left(2,1 ; \mathcal{O}_{k}\right)$ with $k=\boldsymbol{Q}(\sqrt{-1}), \mathcal{O}_{k}$ is the ring of integers in $k$.

Generally the description of the Shimura curve is difficult, and the above two results are nice. But still it remains the following questions.

(1) Is it possible to get more explicit descrition of the Shimura curve ? We are wishing to have a explicit algebraic curves corresponding to the point on the Shimura curve. In this case what does it mean our coordinates, in other words what is our embient space?

(2) Is it possible to get more precise and more simple description of the modular group $G$ ?

(3) Can we describe the period differential equation for our algebraic curves ?

(4) May we find a direct relation between the Gauss hypergeometric differential equation and our modular group $G$ ?

(5) Is it possible to give an explicit "Fourier expansion" for our modular form defined on $\boldsymbol{H}$ with respect to the modular group $G$ ?

In this paper we answer for these questions. We use the Picard modular forms for $k=\boldsymbol{Q}(\sqrt{-3})$ as our main tool.

2000 Mathematics Subjects, Primary: 11G18, 14 G35.

Keywords: Shimura curves, Picard modular forms, hypergeometric functions, triangle groups.

running head: Shimura curve interpretation

This research was supported by Grant-in-Aid for Scientific Research (No. 20540007), Japan Society for the Promotion of Science. 


\section{General tactics}

We set the complex two dimensional hyper ball

$$
\mathscr{D}=\left\{\eta=\left[\eta_{0}: \eta_{1}: \eta_{2}\right] \in \boldsymbol{P}^{2}:{ }^{t} \eta H \bar{\eta}<0\right\}=\left\{(u, v) \in \mathbb{C}^{2}: 2 \operatorname{Re}(\mathrm{v})+|\mathrm{u}|^{2}<0\right\},
$$

here we put $H=\left(\begin{array}{lll}0 & 1 & 0 \\ 1 & 0 & 0 \\ 0 & 0 & 1\end{array}\right), v=\eta_{1} / \eta_{0}$ and $u=\eta_{2} / \eta_{0}$. We set the Picard modular group

$$
\Gamma=\left\{g \in \mathrm{GL}_{3}(\boldsymbol{Z}[\omega]):{ }^{t} \bar{g} H g=H\right\} \text { with } \omega=e^{2 \pi i / 3} .
$$

The element $g=\left(\begin{array}{lll}p_{1} & q_{1} & r_{1} \\ p_{2} & q_{2} & r_{2} \\ p_{3} & q_{3} & r_{3}\end{array}\right) \in \Gamma$ acts on $\mathscr{D}$ by

$$
g(u, v)=\left(\frac{p_{3}+q_{3} v+r_{3} u}{p_{1}+q_{1} v+r_{1} u}, \frac{p_{2}+q_{2} v+r_{2} u}{p_{1}+q_{1} v+r_{1} u}\right) .
$$

For our study of the Shimura curve, we use the hyperball $\mathscr{D}$ together with the group $\Gamma$ or its congruence subgroup. Our domain $\mathscr{D}$ is a coarse moduli space of the family of Picard curves:

$$
C(\lambda): w^{3}=z(z-1)\left(z-\lambda_{1}\right)\left(z-\lambda_{2}\right) .
$$

We shall give a Shimura curve by taking a special hyperplane section, we call it a $k$-disc of $\mathscr{D}$. So we need two fundamental tools, the theory of Picard modular forms and the theory of ball quotient geometry.

\section{The Picard modular form revisited}

Set

$$
\Lambda=\left\{\left(\lambda_{1}, \lambda_{2}\right) \in C^{2}: \lambda_{1} \lambda_{2}\left(\lambda_{1}-1\right)\left(\lambda_{2}-1\right)\left(\lambda_{1}-\lambda_{2}\right) \neq 0\right\} .
$$

The Picard curve is an algebraic curve given by

$$
C(\lambda): w^{3}=z(z-1)\left(z-\lambda_{1}\right)\left(z-\lambda_{2}\right)
$$

for $\lambda \in \Lambda$. It is a curve of genus three and is a three sheeted branched covering over the complex $z$ plane. The Jacobian variety $\operatorname{Jac}(C(\lambda))$ of $C(\lambda)$ has a generalized complex multiplication by $\sqrt{-3}$ of type $(2,1)$. In fact, we have a basis system of holomorphic differentials given by

$$
\varphi=\varphi_{1}=\frac{d z}{w}, \quad \varphi_{2}=\frac{d z}{w^{2}}, \quad \varphi_{3}=\frac{z d z}{w^{2}} .
$$

For the moment, we assume $0<\lambda_{1}<\lambda_{2}<1$. Under this condition we choose the symplectic basis $\left\{A_{1}, \ldots, B_{3}\right\}$ of $H_{1}(C, \mathbb{Z})$ described in Figure 1, that is already used in [Sig1]. Here we put cut lines starting from branch points in the lower half $z$-plane to get simply connected sheets. The real line (resp. dotted line, chained line) indicates an arc on the first sheet (resp. second sheet, third sheet).

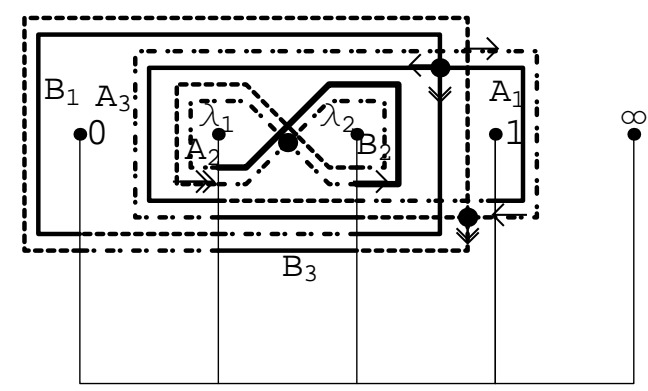


Figure 1. homology basis

Setting $\rho(z, w)=(z, \omega w)$, we have

$$
B_{3}=\rho\left(B_{1}\right), \quad A_{3}=-\rho^{2}\left(A_{1}\right), \quad B_{2}=-\rho^{2}\left(A_{2}\right),
$$

where $\omega$ stands for $\exp [2 \pi \sqrt{-1} / 3]$. We have $A_{i} B_{j}=\delta_{i j}(i, j \in\{1,2,3\})$. Put

$$
\eta_{0}=\int_{A_{1}} \varphi, \quad \eta_{1}=-\int_{B_{3}} \varphi, \quad \eta_{2}=\int_{A_{2}} \varphi .
$$

By the analytic continuation, they are multivalued analytic functions on the domain $\Lambda$. It holds

$$
\left(\begin{array}{l}
\eta_{0} \\
\eta_{1} \\
\eta_{2}
\end{array}\right)=\left(\begin{array}{c}
\int_{A_{1}} \varphi_{1} \\
-\omega^{2} \int_{B_{1}} \varphi_{1} \\
\int_{A_{2}} \varphi_{1}
\end{array}\right)=\left(\begin{array}{c}
-\omega^{2} \int_{A_{3}} \varphi_{1} \\
-\int_{B_{3}} \varphi_{1} \\
-\omega^{2} \int_{B_{2}} \varphi_{1}
\end{array}\right), \quad\left(\begin{array}{c}
\int_{A_{1}} \varphi_{i} \\
-\omega \int_{B_{1}} \varphi_{i} \\
\int_{A_{2}} \varphi_{i}
\end{array}\right)=\left(\begin{array}{c}
-\omega \int_{A_{3}} \varphi_{i} \\
-\int_{B_{3}} \varphi_{i} \\
-\omega \int_{B_{2}} \varphi_{i}
\end{array}\right) \quad(i=2,3)
$$

Set

$$
\Omega_{1}=\left(\int_{A_{j}} \varphi_{i}\right), \quad \Omega_{2}=\left(\int_{B_{j}} \varphi_{i}\right), \quad(1 \leq i, j \leq 3) .
$$

The normalized period matrix of $C(\xi)$ is given by $\Omega=\Omega_{1}^{-1} \Omega_{2}$. By the relations of periods $(2.3)$ together with the symmetricity ${ }^{t} \Omega=\Omega$, we can rewrite

$$
\Omega=\Omega_{1}^{-1} \Omega_{2}=\left(\begin{array}{ccc}
\frac{u^{2}+2 \omega^{2} v}{1-\omega} & \omega^{2} u & \frac{\omega u^{2}-\omega^{2} v}{1-\omega} \\
\omega^{2} u & -\omega^{2} & u \\
\frac{\omega u^{2}-\omega^{2} v}{1-\omega} & u & \frac{\omega^{2} u^{2}+2 \omega^{2} v}{1-\omega}
\end{array}\right),
$$

here we put $u=\frac{\eta_{2}}{\eta_{0}}, v=\frac{\eta_{1}}{\eta_{0}}$. So we set $\Omega=\Omega(u, v)$. The Riemann period relation $\operatorname{Im} \Omega>0$ induces the inequality $2 \operatorname{Re}(v)+|u|^{2}<0$. We set

$$
\mathscr{D}=\left\{\eta=\left[\eta_{0}: \eta_{1}: \eta_{2}\right] \in \boldsymbol{P}^{2}: \eta H^{t} \bar{\eta}<0\right\}=\left\{(u, v) \in \mathbb{C}^{2}: 2 \operatorname{Re}(v)+|u|^{2}<0\right\}
$$

where we put $H=\left(\begin{array}{lll}0 & 1 & 0 \\ 1 & 0 & 0 \\ 0 & 0 & 1\end{array}\right)$. We define our period map $\Phi: \Lambda \rightarrow \mathscr{D}$ by

$$
\Phi\left(\lambda_{1}, \lambda_{2}\right)=\left[\eta_{0}, \eta_{1}, \eta_{2}\right]
$$

Set the Picard modular group

$$
\Gamma=\left\{g \in \mathrm{GL}_{3}(\boldsymbol{Z}[\omega]):{ }^{t} g H \bar{g}=H\right\}
$$

The element $g=\left(\begin{array}{lll}p_{1} & q_{1} & r_{1} \\ p_{2} & q_{2} & r_{2} \\ p_{3} & q_{3} & r_{3}\end{array}\right) \in \Gamma$ acts on $\mathscr{D}$ by

$$
g(u, v)=\left(\frac{p_{3}+q_{3} v+r_{3} u}{p_{1}+q_{1} v+r_{1} u}, \frac{p_{2}+q_{2} v+r_{2} u}{p_{1}+q_{1} v+r_{1} u}\right) .
$$

Let us denote the congruence subgroup $\left\{g \in \Gamma: g \equiv I_{3} \bmod \sqrt{-3}\right\}$ by $\Gamma(\sqrt{-3})$. Set $\bar{\Gamma}=\Gamma /\left\langle-\omega^{2}\right\rangle$ and set $\overline{\Gamma(\sqrt{-3})}=\Gamma(\sqrt{-3}) /\langle\omega\rangle$. We have $\bar{\Gamma} / \overline{\Gamma(\sqrt{-3})} \cong S_{4}$, the symmetric group of degree 4 .

We use the following Riemann theta constants and their Fourier expansions (see [Sig1], p.327):

$$
\vartheta_{k}(u, v)=\vartheta\left[\begin{array}{ccc}
0 & 1 / 6 & 0 \\
k / 3 & 1 / 6 & k / 3
\end{array}\right](0, \Omega(u, v))=\sum_{\mu \in \boldsymbol{Z}[\omega]} \omega^{2 k \operatorname{tr} \mu} H(\mu u) q^{\mathrm{N}(\mu)}
$$


with an index $k \in \boldsymbol{Z}$, where $\operatorname{tr} \mu=\mu+\bar{\mu}, \mathrm{N}(\mu)=\mu \bar{\mu}$ and

$$
H(u)=\exp \left[\frac{\pi}{\sqrt{3}} u^{2}\right] \vartheta\left[\begin{array}{l}
1 / 6 \\
1 / 6
\end{array}\right]\left(u,-\omega^{2}\right), \quad q=\exp \left[\frac{2 \pi}{\sqrt{3}} v\right] .
$$

Apparently it holds $\vartheta_{k}(u, v)=\vartheta_{k+3}(u, v)$, so $k$ runs over $\{0,1,2\}=\boldsymbol{Z} / 3 \boldsymbol{Z}$.

The following properties are already established.

Fact 2.1. $\quad$ (i) ( [Sig1] p.349) The period map $\Phi$ induces a biholomorphic isomorphism from $\xi$-space $\mathbb{P}^{2}(\mathbb{C})$ to the Satake compactification $\overline{\mathscr{D} / \Gamma(\sqrt{-3})}$ of $\mathscr{D} / \Gamma(\sqrt{-3})$. This compactification is obtained by atatching 4 boundary points corresponding to 4 points $\left[\xi_{0}, \xi_{1}, \xi_{2}\right]=[0,0,1],[0,1,0],[1,0,0],[1,1,1]$. We have an action of the $S_{4}$ that is composed of projective linear transformations which causes a permutation of above 4 points on $\boldsymbol{P}^{2}$.

(ii) The following theorem is due to M. Namba [Nam].

Theorem. Let $C(\lambda)$ and $C\left(\lambda^{\prime}\right)$ be two Picard curves. They are isomorphic as Riemann surfaces if and only if we have an automorphism $f$ of $\boldsymbol{C}$ such that $f\left(\left\{0,1, \lambda_{1}, \lambda_{2}\right\}\right)=\left\{0,1, \lambda_{1}^{\prime}, \lambda_{2}^{\prime}\right\}$.

So $\boldsymbol{P}^{2} / S_{4} \cong(\overline{\mathscr{D} / \Gamma(\sqrt{-3})}) / S_{4}=(\mathscr{D} / \Gamma)^{\circ}$ is the moduli space of our family of Picard curves, here ${ }^{\circ}$ means the one point compactification.

(iii) ( [Sig1] p.327) The map $\Theta: \mathscr{D} \longrightarrow \boldsymbol{P}^{2}$ defined by

$$
\Theta\left(\left[\eta_{0}, \eta_{1}, \eta_{2}\right]\right)=\left[\vartheta_{0}(u, v)^{3}, \vartheta_{1}(u, v)^{3}, \vartheta_{2}(u, v)^{3}\right]
$$

gives the inverse of the period map $\Phi$.

(iv) ([Sig1] p.329) The group $\overline{\Gamma(\sqrt{-3})}$ is generated by the classes of

$$
\begin{aligned}
g_{1}=\left(\begin{array}{lll}
1 & 0 & 0 \\
0 & 1 & 0 \\
0 & 0 & \omega
\end{array}\right), g_{2} & =\left(\begin{array}{ccc}
1 & 0 & 0 \\
\omega-\omega^{2} & 1 & 0 \\
0 & 0 & 1
\end{array}\right), g_{3} & =\left(\begin{array}{ccc}
1 & 0 & 0 \\
\omega-1 & 1 & \omega-1 \\
1-\omega^{2} & 0 & 1
\end{array}\right), \\
g_{4} & =\left(\begin{array}{ccc}
1 & \omega-\omega^{2} & 0 \\
0 & 1 & 0 \\
0 & 0 & 1
\end{array}\right), g_{5} & =\left(\begin{array}{ccc}
1 & \omega-1 & \omega-1 \\
0 & 1 & 0 \\
0 & 1-\omega^{2} & 1
\end{array}\right) .
\end{aligned}
$$

This is the projective monodromy group of the multivalued map $\Phi: \Lambda \rightarrow \mathscr{D}$.

(v) ([Sig1] p.346) We have the automorphic property:

$$
\vartheta_{k}(g(u, v))^{3}=\left(p_{1}+q_{1} v+r_{1} u\right)^{3} \vartheta_{k}(u, v)^{3}
$$

for $g=\left(\begin{array}{lll}p_{1} & q_{1} & r_{1} \\ p_{2} & q_{2} & r_{2} \\ p_{3} & q_{3} & r_{3}\end{array}\right) \in \Gamma(\sqrt{-3})$. The sytem $\left\{\vartheta_{k}(g(u, v))^{3}\right\}_{k=0,1,2}$ is a basis of the vector space of automorphic forms with the property (2.9).

(vi) The system of periods $\left\{\eta_{0}, \eta_{1}, \eta_{2}\right\}$ is a basis of the space of solutions for the Appel hypergeometric differential equation $E_{1}\left(a, b, b^{\prime}, c\right)$ with $\left(a, b, b^{\prime}, c\right)=\left(\frac{1}{3}, \frac{1}{3}, \frac{1}{3}, 1\right)$ :

$$
E_{1}\left(a, b, b^{\prime}, c\right):\left\{\begin{array}{l}
r(1-x) x+p(c-(1+a+b) x)-b q y+s(1-x) y-a b z=0 \\
-\left(b^{\prime} p x\right)+s x(1-y)+t(1-y) y+q\left(c-\left(1+a+b^{\prime}\right) y\right)-a b^{\prime} z=0,
\end{array}\right.
$$

with $r=z_{x x}, s=z_{x y}, t=z_{y y}, p=z_{x}, q=z_{y}$. It has singularities along $\boldsymbol{P}^{2}-\Lambda . \overline{\Gamma(\sqrt{-3})}$ is the projective monodromy group of $E_{1}\left(\frac{1}{3}, \frac{1}{3}, \frac{1}{3}, 1\right)$ also. Here we used $(x, y)$ in stead of $\left(\lambda_{1}, \lambda_{2}\right)$. 


\section{Ball quotient geometry}

We sum up from the works of Petkova and Holzapfel just necessary things for our argument.

Set $k=\boldsymbol{Q}(\sqrt{-3})$, and let $\mathcal{O}_{k}$ be the ring of algebraic intergers of $k$. Let $\Gamma^{\prime}$ be an finite index subgroup of $\Gamma=\left\{g \in G L\left(3, \mathcal{O}_{k}\right):{ }^{t} g H \bar{g}=H\right\}$. Let $\mathscr{D}$ be the domain defined in (2.5), and we use the same matrix $H$ used there. We set $\left\langle\eta, \eta^{\prime}\right\rangle_{H}=\eta H^{\bar{t} \eta^{\prime}}$ for $\eta, \eta^{\prime} \in C^{3}$, here ${ }^{-}$indicates the complex conjugate. For a generic point $\eta \in \mathscr{D}$, it corresponds to a Picard curve $C(\lambda)$ with $\lambda=\Theta(\eta)$. We set $A(\lambda)=\operatorname{Jac}(C(\lambda))$.

Let $c \in \mathcal{O}_{k}^{3}$ be a fixed vector with $\langle c, c\rangle_{H}>0$. We call

$$
D_{c}=\left\{\eta \in \mathscr{D}:\langle\eta, c\rangle_{H}=0\right\}
$$

a $k$-disc in $\mathscr{D}$.

The period matrix of $C(\lambda)=\left(\Omega_{1}, \Omega_{2}\right)$ takes the form

$$
M(u, v, \boldsymbol{b}, \boldsymbol{c})=\left(\begin{array}{cccccc}
1 & u & -\omega u & -\omega v & -\omega u & -v \\
b_{0} & b_{2} & -\omega^{2} b_{0} & -\omega^{2} b_{1} & -\omega^{2} b_{2} & -b_{1} \\
c_{0} & c_{2} & -\omega^{2} c_{0} & -\omega^{2} c_{1} & -\omega^{2} c_{2} & -c_{1}
\end{array}\right),
$$

with $\boldsymbol{b}=\left(b_{0}, b_{1}, b_{2}\right), \boldsymbol{c}=\left(c_{0}, c_{1}, c_{2}\right)$. According to the Riemann period relation, we have

$$
\left\langle(1, v, u),\left(b_{0}, b_{1}, b_{2}\right)\right\rangle_{H}=0,\left\langle(1, v, u),\left(c_{0}, c_{1}, c_{2}\right)\right\rangle_{H}=0 .
$$

So we have the period matrix of the form

$$
M(u, v,(1,-\bar{v}, 0),(0,-\bar{u}, 1)) .
$$

This is a "semi-period" owing to the terminology in ([Hol1] section 6). Hence we can apply all the arguments there.

Proposition 3.1. (Essentially due to Holzapfel [Hol1]) Let $D_{c}$ be a $k$-disc. Then for a point $\eta \in D_{c}$, the corresponding Jacobi variety $A(\lambda)$ is isogenous to a product type abelian variety $E_{0} \times A^{\prime}(\lambda)$, with $E_{0}=\boldsymbol{C} /(\boldsymbol{Z}+\omega \boldsymbol{Z})$ and a two dimensional abelian variety $A^{\prime}$.

Proof: According to [Hol1], Cor. 6.23 the abelian variety corresponding to $\eta \in D_{c}$ is isogenous to one of the following types varieties $E_{0} \times S, E_{0} \times E_{\tau}^{2}, E_{0} \times E_{\sigma}^{2}, E_{0}^{3}$. Here $S$ is a simple abelian surface with $Q=\operatorname{End}_{0}(S), Q$ indefinite division quaternion algebra, $E_{\sigma}=\boldsymbol{C} /(\boldsymbol{Z}+\sigma \boldsymbol{Z})$ is an elliptic curve with imaginary quadratic multiplication by $\boldsymbol{Q}(\sigma)$ and $E_{\tau}=\boldsymbol{C} /(\boldsymbol{Z}+\tau \boldsymbol{Z})$ is an elliptic curve not of CM-type.

q.e.d.

Proposition 3.2. The $k$-disc $D_{c}$ is a Shimura variety for the quaternion algebra $B=\left(\frac{-3,\langle c, c\rangle_{H}}{Q}\right)$. Namely, it is a one dimensional coarse moduli space of the abelian surfaces $A^{\prime}$ for which generically we have

$$
B=\operatorname{End}_{0}\left(A^{\prime}\right)
$$

Proof. Let $p: \mathscr{D} \rightarrow \mathscr{D} / \Gamma$ be the natural projection. From [Hol2], section 4.4., we know that $\mathscr{D} / \Gamma$ is a Picard modular surface and $D_{c} / \Gamma=p\left(D_{c}\right) \subset \mathscr{D} / \Gamma$ is an algebraic curve. The points of $\mathscr{D} / \Gamma$ parametrize isomorphy classes of abelian threefolds $A(\lambda)$ with $k$-multiplication and those of $D_{c} / \Gamma$ correspond to the abelian varieties of type $E_{0} \times A^{\prime}(\lambda)$, Prop. 3.1, i.e. parametrize abelian surfaces $A^{\prime}(\lambda)$.

Consider the group $\Gamma_{c}=\left\{\gamma \in \Gamma: \gamma D_{c}=D_{c}\right\}$ which is isomorphic to $U((1,1), \mathcal{O})$ and $G_{c}=\Gamma_{c} /\{\gamma \in$ $\left.\Gamma: \gamma_{\mid D_{c}}=\operatorname{id}_{D_{c}}\right\}$, then $D_{c} / \Gamma_{c}=D_{c} / G_{c}$ is an algebraic curve, which is a normalization of $D_{c} / \Gamma$, [Hol2]. The period lattice of the abelian surface $A^{\prime}(\lambda)$, corresponding to $\eta \in D_{c}$, is isomorphic to a sublattice of rank 4 of the period lattice of $A(\lambda)$, [Hol1] section 6. For $\lambda=\gamma\left(\lambda^{\prime}\right)$ the abelian varieties $A(\lambda)$ and $A\left(\lambda^{\prime}\right)$ 
are isomorphic, and so are $A^{\prime}(\lambda)$ and $A^{\prime}\left(\lambda^{\prime}\right)$ too. By construction the quotient $D_{c} / \Gamma_{c}$ is a Picard modular curve and as such parametrizes abelian surfaces with $k$-multiplication [Shi2], hence it parametrizes the isomorphy classes of abelian surfaces $A^{\prime}(\lambda)$. Furthermore, following [Shi2] Prop. 18, for a generic $\eta \in D_{c}$, the corresponding abelian surface $A^{\prime}(\lambda)$ has also a multiplication by an indefinite quaternion algebra $B$, i.e. there exists an embedding $B \subseteq \operatorname{End}_{0}\left(A^{\prime}(\lambda)\right)$. According to [Pet] section 6.5 the quaternion algebra $B$ is then isomorphic to $\left(\frac{-3,\langle c, c\rangle_{H}}{Q}\right)$.

Remark 3.1. We put a direct proof of the above two propositions in Section 6. Moreover we shall show the equality $\operatorname{End}\left(A^{\prime}(\lambda)\right) \cong \mathcal{O}_{B}$, where $\mathcal{O}_{B}$ is the unique (up to conjugacy) maximal order of $B$.

\section{Results}

Set $\boldsymbol{c}=(1,1,0)$. Its $H$-norm $\boldsymbol{c} H^{t} \bar{c}$ is 2 . Its $H$-orthogonal complement is given by

$$
\boldsymbol{c}^{\perp}=\left\{\left[\eta_{0}, \eta_{1}, \eta_{2}\right] \in \boldsymbol{P}^{2}: \eta_{0}+\eta_{1}=0\right\} .
$$

Set $D_{\boldsymbol{c}}=\{(u, v) \in \mathscr{D}: v=-1\}=\boldsymbol{c}^{\perp} \cap \mathscr{D}$ and

$$
\Gamma_{c}=\left\{g \in \Gamma: g\left(D_{c}\right)=D_{c}\right\} /\left\{g \in \Gamma: g_{\mid D_{c}}=\operatorname{id}_{D_{c}}\right\} .
$$

According to Petkova and Holzapfel ([Pet], [Hol3]) we know that $D_{c} / \Gamma_{c}$ is the Shimura curve for

$$
B=\left(\frac{-3,\|\boldsymbol{c}\|_{H}}{\boldsymbol{Q}}\right)=\left(\frac{-3,2}{\boldsymbol{Q}}\right)
$$

with $\operatorname{Disc}(B)=6$. (We have

$$
\operatorname{Disc}(B)=\prod_{(-3,2)_{p}=-1} p=2 \cdot 3=6 .
$$

)

So this is the case discussed in [Voi] and also studied in [Pet].

Theorem 4.1. Set a complex line $L_{\boldsymbol{c}}=\left\{\lambda_{1}+\lambda_{2}=1\right\}$ in $\left(\lambda_{1}, \lambda_{2}\right)$ space $\boldsymbol{P}^{2}$. Then we have

$$
\Theta\left(D_{\boldsymbol{c}}\right)=L_{\boldsymbol{c}}
$$

Namely our Shimura curve is realized as a hyperplane section in the vector space space $\left\langle\vartheta_{0}^{3}, \vartheta_{1}^{3}, \vartheta_{2}^{3}\right\rangle$ of Picard modular forms.

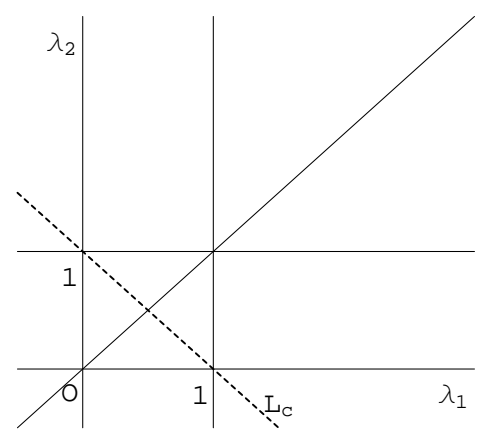

Figure of the Shimura curve $L_{c}$

Theorem 4.2. For a Picard curve $C(\lambda): w^{3}=z(z-1)\left(z-\lambda_{1}\right)\left(z-\lambda_{2}\right)$ with $\lambda_{1}+\lambda_{2}=1$, we have a decomposition

$$
J(C(\lambda)) \cong E_{0} \times A^{\prime}(\lambda) \quad(\text { up to isogeny }),
$$


where $E_{0}=\boldsymbol{C} /(\omega \boldsymbol{Z}+\boldsymbol{Z})$ and $A^{\prime}(\lambda)$ is a 2-dimentional abelian variety. And generically we have

$$
\operatorname{End}_{0}\left(A^{\prime}(\lambda)\right)=B
$$

Namely $C(\lambda)$ with $\lambda_{1}+\lambda_{2}=1$ is the corresponding curve for our Shimura variety.

Theorem 4.3. Put $\lambda_{1}=\frac{1}{2}(1+s), \lambda_{2}=\frac{1}{2}(1-s)$. On the line $L_{c}$ the Appell differential equation $E\left(\frac{1}{3}, \frac{1}{3}, \frac{1}{3}, 1\right)$ reduces to

$$
\begin{array}{r}
27 s^{2}\left(-1+s^{2}\right)^{2}\left(3+s^{2}\right) z_{s s s}+18 s\left(3-38 s^{2}+27 s^{4}+8 s^{6}\right) z_{s s} \\
+6\left(-9-60 s^{2}+127 s^{4}+22 s^{6}\right) z_{s}+8 s^{3}\left(9+s^{2}\right) z=0 .
\end{array}
$$

Remark 4.1. This is a Fuchsian differential equation of rank 3. Looking at (4.1) we know that it has new singularities $u= \pm \sqrt{-3}$ other than the expected singularities $L_{\boldsymbol{c}} \cap\left(\boldsymbol{P}^{2}-\Lambda\right)=\{0, \pm 1, \infty\}$. We have the Riemann scheme of (4.1):

$$
\left\{\begin{array}{cccccc}
0 & 1 & -1 & \sqrt{-3} & -\sqrt{-3} & \infty \\
0 & 0 & 0 & 0 & 0 & \frac{1}{3} \\
2 & \frac{1}{3} & \frac{1}{3} & 1 & 1 & \frac{2}{3} \\
\frac{1}{3} & -\frac{2}{3} & -\frac{2}{3} & 3 & 3 & \frac{4}{3}
\end{array}\right\} .
$$

So $u= \pm \sqrt{-3}$ are apparent singularities.

Let

$$
F_{1}\left(\lambda_{1}, \lambda_{2}\right)=F_{1}\left(\frac{1}{3}, \frac{1}{3}, \frac{1}{3}, 1 ; \lambda_{1}, \lambda_{2}\right)=1+\sum_{m+n>0} \frac{\left(\frac{1}{3}, m+n\right)\left(\frac{1}{3}, m\right)\left(\frac{1}{3}, n\right)}{(1, m+n) m ! n !} \lambda_{1}^{m} \lambda_{2}^{n}
$$

be the Appell hypergeometric series that is a solution of $E\left(\frac{1}{3}, \frac{1}{3}, \frac{1}{3}, 1\right)$.

Theorem 4.4. Let $f(s)=F_{1}\left(\frac{1}{2}(1+s), \frac{1}{2}(1-s)\right)$ be the restriction of $F_{1}$ on $L_{c}$. Then $f(s)$ is an even function of $s$. So we put $f(s)=g(t)$ with $t=s^{2}$. In this situation $g(t)$ satisfies the following Gauss hypergeometric differential equation:

$$
g^{\prime \prime}(t)+\frac{9 t-5}{6 t(t-1)} g^{\prime}(t)+\frac{1}{18 t(t-1)} g(t)=0 .
$$

Remark 4.2. (1) This theorem shows that the system (4.1) contains the subsystem (4.2) of rank 2. And it corresponds to the fact that we have the linear relation $\eta_{0}+\eta_{1}=0$ in the period domain $\mathscr{D}$.

(2) The Riemann scheme of (4.2) is

$$
\left\{\begin{array}{ccc}
0 & 1 & \infty \\
0 & 0 & \frac{1}{6} \\
\frac{1}{3} & \frac{1}{6} & \frac{1}{3}
\end{array}\right\} .
$$

So (4.2) is the Gauss hypergeometric differential equation $E\left(\frac{1}{6}, \frac{1}{3}, \frac{2}{3}\right)$, and its monodromy group is the triangle group $\Delta(3,6,6)$. We can find it in the list of arithmetic co-compact triangle groups by K. Takeuchi [Tak].

Definition 4.1. Set $B=\left(\frac{a, b}{Q}\right)$ be an indefinite quaternion algebra over $\boldsymbol{Q}$. We say $S_{B}$ is a Shimura curve for $B$, if it is a moduli space of the isomorphism classes of principally polarized Abelian surfaces with the condition $B \cong \operatorname{End}_{0}(A)$ for generic members. 
Theorem 4.5. Set $\overline{\Gamma_{c}}=\left\{g \in \bar{\Gamma}: g\left(D_{c}\right)=D_{c}\right\} /\left\{g \in \bar{\Gamma}: g_{\mid D_{c}}=i d_{D_{c}}\right\}$. Under the identification induced from the isomorphism $\Theta: \overline{D / \Gamma(\sqrt{-3})} \rightarrow \boldsymbol{P}^{2}$, we have the representation of the Shimura curve:

$$
S_{B}=L_{\boldsymbol{c}} /\langle\sigma\rangle \cong D_{\boldsymbol{c}} / \overline{\Gamma_{c}} \cong \boldsymbol{H} / \Delta(3,6,6) .
$$

Remark 4.3. The Shimura curve for a quaternion algebra B might be considered to be the moduli space for the family of isomorphism classes of (principally polarized) abelian varieties A with the endomorphism structure $\operatorname{End}_{0}(A)=B$ for generic members. On the other hand, normally Shimura curve is defined as the quotient space of $\boldsymbol{H}$ by the norm 1 group $\Gamma^{B}(1)$ induced from the maximal order of $B$, or by its some finite index extension. As far as the authors know, there is no discussion about the exact relation of these two (or three) definitions.

Our Shimura curve $L_{\boldsymbol{c}} /\langle\sigma\rangle=\mathrm{H} / \Delta(3,6,6)$ is the exact moduli space in the above sense. The inverse Schwarz map for the Gauss hypergeometric differential equation (4.2) is described via theta map $\Theta_{\mid D_{c}}$ in a completely explicit way.

The authors discovered the relation in Theorem 3.1 by computer aided experiments using this $\Theta$ representation.

\section{Proofs}

\subsection{Proof of Theorem 4.1}

Set

$$
C(\lambda): w^{3}=z(z-1)\left(z-\lambda_{1}\right)\left(z-\lambda_{2}\right)
$$

with $0<\lambda_{1}, \lambda_{2}<1$. Suppose we have

$$
\lambda_{1}+\lambda_{2}=1 .
$$

We have the canonical basis $\left\{A_{1}, \ldots, B_{3}\right\}$ of $H_{1}(C(\lambda, \boldsymbol{Z}))$ described in the fugure 1 . We defined the period map $\Phi$ by setting

$$
\Phi\left(\lambda_{1}, \lambda_{2}\right)=\left[\eta_{0}, \eta_{1}, \eta_{2}\right]:=\left[\int_{A_{1}} \frac{d z}{w},-\omega^{2} \int_{B_{1}} \frac{d z}{w}, \int_{A_{2}} \frac{d z}{w}\right] .
$$

We claim that we have

$$
\eta_{0}+\eta_{1}=0 .
$$

Note that we have the automorphism $\sigma:(z, w) \mapsto\left(z^{\prime}, w^{\prime}\right):=(1-z, w)$ of $C(\lambda)$. The homology cycle $A_{1}\left(A_{3}\right.$, resp.) is transported to $-B_{3}\left(B_{1}\right.$, resp.) (see figure 2$)$ by $\sigma$.
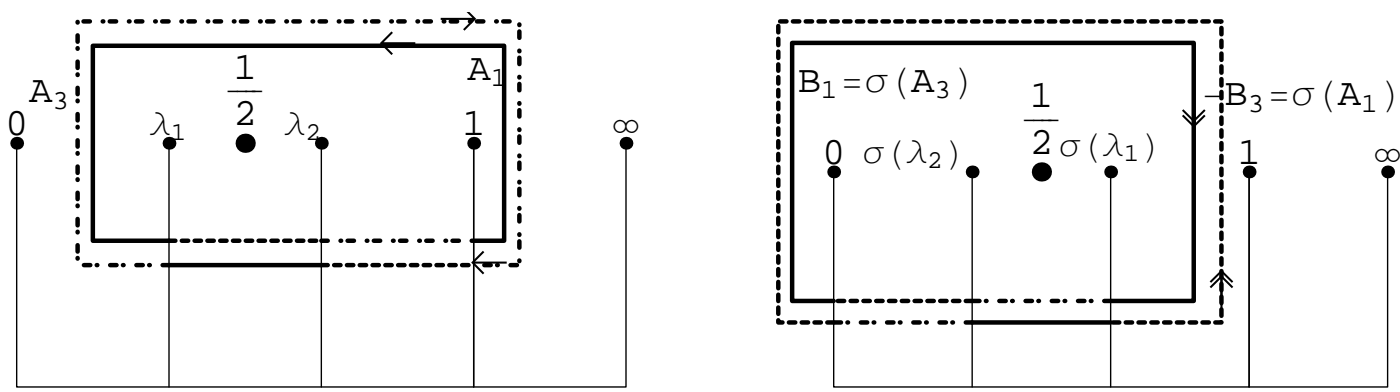

Figure 2 
By the same way we have

$$
\sigma\left(\begin{array}{l}
A_{1} \\
A_{2} \\
A_{3} \\
B_{1} \\
B_{2} \\
B_{3}
\end{array}\right)=\left(\begin{array}{cccccc}
0 & 0 & 0 & 0 & 0 & -1 \\
0 & 0 & 0 & 0 & -1 & 0 \\
0 & 0 & 0 & 1 & 0 & 0 \\
0 & 0 & 1 & 0 & 0 & 0 \\
0 & -1 & 0 & 0 & 0 & 0 \\
-1 & 0 & 0 & 0 & 0 & 0
\end{array}\right)\left(\begin{array}{c}
A_{1} \\
A_{2} \\
A_{3} \\
B_{1} \\
B_{2} \\
B_{3}
\end{array}\right) .
$$

It holds

$$
\int_{\sigma\left(A_{1}\right)} \varphi=\int_{A_{1}} \sigma^{*}(\varphi) .
$$

We have

$$
\int_{\sigma\left(A_{1}\right)} \varphi=-\int_{B_{3}} \varphi=-\omega^{2} \int_{B_{1}} \varphi=\eta_{1}
$$

and

$$
\int_{A_{1}} \sigma^{*}(\varphi)=\int_{A_{1}}(-\varphi)=-\eta_{0}
$$

Then we obtain the required assertion.

q.e.d.

Theorem 4.2 is a direct cosequence of Proposition 3.2.

\subsection{Proof of Theorem 4.3}

The equation is obtained by a straight calculation. We perform it in the following way.

In this proof we denote the variables $\lambda_{1}, \lambda_{2}$ by $x, y$, respectively.

1) Start from the system $\left\{L_{1} z=0, L_{2} z=0, L_{3} z=0\right\}$ with

$$
\left\{\begin{array}{l}
L_{1}=x(1-x) D_{x x}+(1-x) y D_{x y}+(c-(a+b+1) x) D_{x}-b y D_{y}-a b \\
L_{2}=x(1-y) D_{x y}+y(1-y) D_{y y}+\left(c-\left(a+b^{\prime}+1\right) y\right) D_{y}-b^{\prime} x D_{x}-a b^{\prime} \\
L_{3}=(x-y) D_{x y}-b^{\prime} D_{x}+b D_{y}
\end{array}\right.
$$

of the Appell hypergeometric differential equation with variables $x, y$. Let $D_{x}, D_{y}$ denote the partial derivative operator, and we use the other higher derivative operators in a similar way.

2) We make the coordinate change

$$
\left\{\begin{array}{l}
\mu=x-y \\
\nu=x+y
\end{array}\right.
$$

We can rewrite the system (5.4) in terms of $\mu, \nu$. We let them denote $\left\{\tilde{L_{1}}, \tilde{L_{2}}, \tilde{L_{3}}\right\}$.

3) We make the partial derivative of the operator $\tilde{L}_{1}$ with respect to $\mu$, and express it by using only $D_{\mu \mu \mu}, D_{\mu \mu}, D_{\mu}$, but it still contains the variable $\nu$. Let it denote by $L_{0, \nu}$. We have

$$
\begin{aligned}
& L_{0, \nu} /(-3+2 \mu+3 \nu)=27 \mu^{2}(\mu-\nu)(2+\mu-\nu)(-2+\mu+\nu)(\mu+\nu)\left(\mu^{2}+6 \nu-3 \nu^{2}\right) D_{\mu \mu \mu} \\
& +18 \mu\left(-12 \mu^{4}+8 \mu^{6}-144 \mu^{2} \nu+78 \mu^{4} \nu+208 \mu^{2} \nu^{2}-39 \mu^{4} \nu^{2}+24 \nu^{3}-136 \mu^{2} \nu^{3}-36 \nu^{4}+34 \mu^{2} \nu^{4}+18 \nu^{5}-3 \nu^{6}\right) D_{\mu \mu} \\
& +6\left(22 \mu^{6}+\left(-216 \mu^{2}+254 \mu^{4}\right) \nu+\left(300 \mu^{2}-127 \mu^{4}\right) \nu^{2}+\left(-72-192 \mu^{2}\right) \nu^{3}+\left(108+48 \mu^{2}\right) \nu^{4}-54 \nu^{5}+9 \nu^{6}\right) D_{\mu} \\
& +8 \mu^{3}\left(\mu^{2}+18 \nu-9 \nu^{2}\right)
\end{aligned}
$$

4) Finally we put $\nu=1$ in $L_{0, \nu}$. Let us denote it by $L_{0}$. This is the required differential operator.

q.e.d.

\subsection{Proof of Theorem 4.4}

By observing the series $f(s)$ we get the required Gauss hypergeometric differential equation. The calculation of the Riemann scheme is straight forward.

q.e.d. 


\subsection{Proof of Theorem 4.5}

Note that the action of the symmetric group $S_{4}$ on $L_{c}$ is nothing but the involution $\sigma: s \mapsto-s$. By the Fact 1.1 (ii), $L_{\boldsymbol{c}} /\langle\sigma\rangle$ is the moduli space of our restricted family of Picard curves

$$
w^{3}=z(z-1)\left(z-\frac{1}{2}(1+s)\right)\left(z-\frac{1}{2}(1-s)\right) .
$$

Under the identification stated in Fact 1.1. (i)(ii) we have $L_{c} /\langle\sigma\rangle \cong D_{c} / \overline{\Gamma_{c}}$. The projective monodromy group of $(4.2)$ is the triangle group $\Delta(3,6,6)$. By the transformation of the variable $D_{c}$ coincides with the upper half plane $\boldsymbol{H}$.

Still more, according to Proposition 3.2 and Theorem 4.2 our Jacobi variety $J a c(C(\lambda))=A(\lambda)$ is isogenous to a product $E_{0} \times A^{\prime}(\lambda)$ with $B \subset \operatorname{End}_{0}\left(A^{\prime}(\lambda)\right)$. In our situation, the isogenous decomposition $E_{0} \times A^{\prime}(\lambda)$ uniquely determines the full period matrix of $C(\lambda)$ (see also (6.4) and (6.5)). By referring the theorem of Namba, $L_{\boldsymbol{c}} /\langle\sigma\rangle$ is considered to be the moduli space of $\left.\{C(\lambda))\right\}$. So we obtain the assertion.

q.e.d.

\section{Direct observation of the endomorphism algebra}

By making up the period matrix of our Picard curve (5.1) with (5.2), we have a direct argument to show the equality

$$
\operatorname{End}\left(A^{\prime}(\lambda)\right)=\mathcal{O}_{B}
$$

where $\mathcal{O}_{B}$ is the maximal order of $B=\left(\frac{-3,2}{\boldsymbol{Q}}\right)$. Note that we have

$$
\begin{aligned}
& B=\boldsymbol{Q}+\boldsymbol{Q} X+\boldsymbol{Q} Y+\boldsymbol{Q} X Y \\
& \mathcal{O}_{B}=\boldsymbol{Z}+\boldsymbol{Z} X^{\prime}+\boldsymbol{Z} Y+\boldsymbol{Z} X^{\prime} Y
\end{aligned}
$$

with

$$
X=\left(\begin{array}{cc}
\sqrt{-3} & 0 \\
0 & -\sqrt{-3}
\end{array}\right), X^{\prime}=\left(\begin{array}{cc}
\omega^{2} & 0 \\
0 & \omega
\end{array}\right), Y=\left(\begin{array}{ll}
0 & 1 \\
2 & 0
\end{array}\right) .
$$

For a general Picard curve (2.1) we set

$$
\left\{\begin{array}{l}
\alpha_{1}=\int_{A_{1}} \varphi_{1}, \alpha_{2}=\int_{A_{2}} \varphi_{1}, \alpha_{3}=\int_{B_{1}} \varphi_{1} \\
\beta_{1}=\int_{A_{1}} \varphi_{2}, \beta_{2}=\int_{A_{2}} \varphi_{2}, \beta_{3}=\int_{B_{1}} \varphi_{2} \\
\gamma_{1}=\int_{A_{1}} \varphi_{3}, \gamma_{2}=\int_{A_{2}} \varphi_{3}, \gamma_{3}=\int_{B_{1}} \varphi_{3}
\end{array}\right.
$$

According to (2.3), we have the full period matrix

$$
\left(\Omega_{1}, \Omega_{2}\right)=\left(\begin{array}{cccccc}
\alpha_{1} & \alpha_{2} & -\omega \alpha_{1} & \alpha_{3} & -\omega \alpha_{2} & \omega^{2} \alpha_{3} \\
\beta_{1} & \beta_{2} & -\omega^{2} \beta_{1} & \beta_{3} & -\omega^{2} \beta_{2} & \omega \beta_{3} \\
\gamma_{1} & \gamma_{2} & -\omega^{2} \gamma_{1} & \gamma_{3} & -\omega^{2} \gamma_{2} & \omega \gamma_{3}
\end{array}\right)
$$

When we have $\lambda \in L_{\boldsymbol{c}}$, it holds $\omega^{2} \alpha_{3}=\alpha_{1}$. In fact

$$
\int_{A_{1}} \varphi_{1}=-\int_{\sigma\left(B_{3}\right)} \varphi_{1}=-\int_{B_{3}} \sigma^{*} \varphi_{1}=\int_{B_{3}} \varphi_{1}=\omega^{2} \int_{B_{1}} \varphi_{1} .
$$

Using the facts $\sigma^{*} \varphi_{2}=-\varphi_{2}, \sigma^{*} \varphi_{3}=\varphi_{3}$ we have

$$
\omega \beta_{3}=\beta_{1}, \omega \gamma_{3}=-\gamma_{1}
$$


In this case it holds

$$
\left(\Omega_{1}, \Omega_{2}\right)=\left(\begin{array}{cccccc}
\alpha_{1} & \alpha_{2} & -\omega \alpha_{1} & \omega \alpha_{1} & -\omega \alpha_{2} & \alpha_{1} \\
\beta_{1} & \beta_{2} & -\omega^{2} \beta_{1} & \omega^{2} \beta_{1} & -\omega^{2} \beta_{2} & \beta_{1} \\
\gamma_{1} & \gamma_{2} & -\omega^{2} \gamma_{1} & -\omega^{2} \gamma_{1} & -\omega^{2} \gamma_{2} & -\gamma_{1}
\end{array}\right)
$$

According to the Riemann bilinear relation, we have

$$
-2 \alpha_{1} \beta_{1}+\alpha_{2} \beta_{2}=0
$$

If we regard (6.2) as a generator system of a lattice in $C^{3}$, it is the same that of

$$
L=\left(\begin{array}{cccccc}
\alpha_{1} & \alpha_{2} & -\omega \alpha_{1} & -\omega \alpha_{2} & 0 & 0 \\
\beta_{1} & \beta_{2} & -\omega^{2} \beta_{1} & -\omega^{2} \beta_{2} & 0 & 0 \\
\gamma_{1} & \gamma_{2} & -\omega^{2} \gamma_{1} & -\omega^{2} \gamma_{2} & 2 \gamma_{1} & -2 \omega^{2} \gamma_{1}
\end{array}\right) .
$$

By putting $\kappa=\alpha_{2} / \alpha_{1}, \mu=\beta_{2} / \beta_{1}$, set

$$
L^{\prime}=\left(\begin{array}{cccc}
1 & \kappa & -\omega & -\omega \kappa \\
1 & \mu & -\omega^{2} & -\omega^{2} \mu
\end{array}\right)
$$

We see directly that $\operatorname{Jac}(C(\lambda))$ has an isogenous decomposition $E_{0} \times A^{\prime}(\lambda)$ with

$$
A^{\prime}(\lambda)=C^{2} / L^{\prime}
$$

By ( 6.3) we have

$$
L^{\prime}=\left(\begin{array}{cccc}
1 & \kappa & -\omega & -\omega \kappa \\
\kappa & 2 & -\omega^{2} \kappa & -2 \omega^{2}
\end{array}\right)
$$

So we have endomorphisms of $\operatorname{End}\left(L^{\prime}\right)$ in the complex representations:

$$
\frac{-1+\sqrt{-3}}{2}=\left(\begin{array}{cc}
\omega^{2} & 0 \\
0 & \omega
\end{array}\right), \sqrt{2}=\left(\begin{array}{ll}
0 & 1 \\
2 & 0
\end{array}\right) \text {. }
$$

That means generically we have (6.1).

\section{References}

[Als] Montserrat Alsina; Pilar Bayer, Quaternion orders, quadratic forms, and Shimura curves, Providence, RI : American Mathematical Society, 2004 (CRM Monograph Series, 22)

[Dza] Amir Dzambic, Geometrie arithmetisch definierter Ballquotientenächen, Dissertation, 2007, Berlin Humboldt Univ.

[Elk] Noam Elkies, Shimura curve computation, Lecture Notes in Computer Science 1423 (proceedings of ANTS-3, 1998; J.P.Buhler, ed.), 1998, pp. 1-47.

[Hol1] Rolf Peter Holzapfel, Hierarchies of Endomorphism Algebras of Abelian Varieties Corresponding to Picard Modular Surfaces, Forschunbsschwehrpunkt Komplexe Mannigfaltigkeiten, Schriftenreihe Heft Nr. 190, 1994.

[Hol2] Rolf Peter Holzapfel, Ball and Surface Arithmetic, Vieweg 1998.

[Hol3] Rolf Peter Holzapfel, A. Pineiro and A. Vladov, Picard-Eisenstein Metrics and Class Fields Connected with Apollonius Cycle, Preprint, Berlin Humboldt Univ., 1999.

[Kur] Akira Kurihara, On some examples of equations defining Shimura curves and the Mumford uniformization, J. Fac. Sci. Univ. Tokyo 25 (1979), 277- 301.

[Nam] Makoto Namba, Equivalence problem and automorphism groups of certain compact Riemann surfaces, Tsukuba J. Math. 5(1981), 319-338.

[Pet] Maria Petkova, Families of Algebraic Curves with Application in Coding Theory and Cryptography, Dissertation, 2009, Berlin Humboldt Univ.

[Sig1] Hironori Shiga, On the representation of the Picard modular function by $\theta$ constants I-II, Pub. R.I.M.S. Kyoto Univ. 24 (1988), 311-360. 
[Shi1] Goro Shimura, Arithmetic of Unitary Groups, The Annals of Mathematics, 2nd Ser., Vol. 79, No. 2. (Mar., 1964), pp. 369-409.

[Shi2] Goro Shimura, On Analytic Families of Polorized Abelian Varieties and Automorphic functions, The Annals of Mathematics, 2nd Ser., Vol. 78, No. 1. (Jul., 1963), pp. 149-192.

[Tak] Kisao Takeuchi, Arithmetic triangle groups, J. Math. Soc. Japan 29(1977), 91-106.

[Voi] John Voight, Shimura curve computations, Publication of Clay Math. Inst. Summer School, 2006.

Maria Petkova

Departement Mathematik

ETH Zurich

Rämistrasse 1018092 Zurich Switzerland

E-Mail: maria.petkova@math.ethz.ch

Hironori Shiga (Corresponding author)

Department of Mathematical Sciences, Institute of Science and Engineering

Waseda University

Shinjuku-ku Ohkubo 3-4-1, Tokyo 169-8555 Japan

E-Mail: shiga@math.s.chiba-u.ac.jp 